\title{
Avaliação de conhecimento e condutas clínicas na prática profissional farmacêutica
}

\author{
Evaluation of knowledge and clinical conduct in pharmaceutical professional practice \\ Evaluación del conocimiento y la conducta clínica en la práctica profesional farmacéutica
}

Karícia Lima de Freitas Bonfim ORCID: https://orcid.org/0000-0002-9805-6883 Universidade Federal do Piauí, Brasil E-mail: karicia_freitas@hotmail.com Thercyo Ariell Costa Pereira ORCID: https://orcid.org/0000-0003-4834-7479 Centro Universitário UNIFACID, Brasil E-mail: thercimc@gmail.com

Kellyanne Soares de Sousa ORCID: https://orcid.org/0000-0002-3784-7155 Centro Universitário UNIFACID, Brasil E-mail: kellyannesousa03@gmail.com Manoela Cavalcante Ribeiro ORCID: https://orcid.org/0000-0003-0785-4810 Centro Universitário UNIFACID, Brasil E-mail: manoelacavalcanteribeiro@hotmail.com

Luiza Helena Nascimento Lopes

ORCID: https://orcid.org/0000-0003-1317-1549 Centro Universitário UNIFACID, Brasil

E-mail: luizalopes06@hotmail.com Davi Portela Bessa

ORCID https://orcid.org/0000-0003-1169-7926 Centro Universitário UNIFACID, Brasil

E-mail: bessadavi1@gmail.com

Joyce Jamylle Dias Borges

ORCID: https://orcid.org/0000-0002-7413-4197 Centro Universitário UNIFACID, Brasil

E-mail: joycejamylle_@hotmail.com

Fernanda Arias de Almeida Macedo ORCID: https://orcid.org/0000-0002-7948-8015 Centro Universitário UNIFACID, Brasil E-mail: ariasfernandinha@gmail.com

Thuany Cristiny Batista Feitosa Carneiro

ORCID: https://orcid.org/0000-0001-9817-249X Centro Universitário UNIFACID, Brasil E-mail: thuany_cristiny@hotmail.com

Edna Nagela da Silva Maciel

ORCID: https://orcid.org/0000-0002-9957-5941 Centro Universitário UNIFACID, Brasil

E-mail:nglmaciel@outlook.com

Luiz Carlos Nogueira Falcão

ORCID: https://orcid.org/0000-0003-0987-5308 Centro Universitário UNIFACID, Brasil E-mail: lcnf@hotmail.com

Fábio Mesquita Camelo

ORCID: https://orcid.org/0000-0002-9153-5507 Centro Universitário UNIFACID, Brasil

E-mail: fabiomesquita_2001@hotmail.com

Alice Lima Rosa Mendes

ORCID: https://orcid.org/0000-0002-1960-9647

Universidade de Brasília, Brasil

E-mail: alice_lima_@hotmail.com

Mayara Ladeira Coêlho

ORCID: https://orcid.org/0000-0002-8084-5964 Centro Universitário UNIFACID, Brasil

Resumo

O farmacêutico comunitário vem ampliando seu escopo de atuação. À dispensação vem sendo acrescida as responsabilidades no sistema de saúde do brasileiro, como a busca para o desenvolvimento de serviços farmacêuticos 
clínicos. Assim, objetivou-se avaliar o conhecimento dos farmacêuticos comunitários atuantes sobre as atribuições clínicas instituídas pela resolução $\mathrm{n}^{\circ}$ 585/13, na cidade de Teresina-Piauí. O trabalho foi realizado através de duas etapas, nas quais foram entrevistados 100 profissionais em cada, todos devidamente registrados no Conselho Regional de Farmácia do Piauí (CRF-PI). As informações foram coletadas e analisadas por meio do programa Microsoft Office Excel ${ }^{\circledR}$ 2013. Os resultados revelaram que $82 \%$ afirmaram conhecer a referida resolução, apenas $10 \%$ citaram atividades que não se enquadravam no conceito de serviço farmacêutico. Sobre o acompanhamento farmacoterapêutico, percebeu-se que a grande maioria tinha conhecimento da atuação $(79 \%)$ e realizam algum tipo de serviço clínico farmacêutico (82\%). Apenas 6\% afirmaram não ter espaço adequado dentro da farmácia/drogaria para realizar esses serviços, $88 \%$ informaram que já fizeram/fazem especialização e somente $3 \%$ declararam realizar a prescrição farmacêutica. Embora a grande maioria dos profissionais farmacêuticos alegarem ter conhecimento da resolução ${ }^{\circ}$ $585 / 2013$, não sabem informar de fato sobre esta resolução. Além disso, destaca-se o baixo percentual de profissionais que realizam prescrições farmacêuticas. Esse contexto demonstra que são necessárias ações educativas que preparem adequadamente esse profissional para atuação clínica.

Palavras-chave: Serviços farmacêuticos; Resolução; Farmácia comunitária.

\begin{abstract}
Community pharmacists have been expanding their scope of action. Dispensing has been added to the responsibilities in the Brazilian health system, such as the search for the development of clinical pharmaceutical services. Thus, the objective was to assess the knowledge of community pharmacists working on the clinical attributions established by Resolution No. 585/13, in the city of Teresina-Piauí. The work was carried out through two stages, in which 100 professionals were interviewed in each, all duly registered with the Regional Council of Pharmacy of Piauí (CRF-PI). The information was collected and analyzed using the Microsoft Office Excel® 2013 program. The results revealed that $82 \%$ claimed to know the aforementioned resolution, only $10 \%$ mentioned activities that did not fit the concept of pharmaceutical service. Regarding the pharmacotherapeutic follow-up, it was noticed that the vast majority were aware of the action (79\%) and performed some type of clinical pharmaceutical service (82\%). Only 6\% said they did not have adequate space within the pharmacy/drugstore to carry out these services, $88 \%$ reported that they have already done/are doing specialization and only $3 \%$ declared that they were taking the pharmaceutical prescription. Although the vast majority of pharmaceutical professionals claim to be aware of resolution No. 585/2013, they do not know how to actually inform about this resolution. In addition, the low percentage of professionals who perform pharmaceutical prescriptions stands out. This context demonstrates that educational actions are needed to adequately prepare these professionals for clinical work.
\end{abstract}

Keywords: Pharmaceutical services; Resolution; Community pharmacy.

\title{
Resumen
}

Los farmacéuticos comunitarios han ido ampliando su ámbito de actuación. La dispensación se ha sumado a las responsabilidades en el sistema de salud brasileño, como la búsqueda para el desarrollo de servicios farmacéuticos clínicos. Así, el objetivo fue evaluar el conocimiento de los farmacéuticos comunitarios que trabajan en las atribuciones clínicas establecidas por la Resolución No. 585/13, en la ciudad de Teresina-Piauí. El trabajo se llevó a cabo a través de dos etapas, en las que se entrevistó a 100 profesionales en cada una, todos debidamente registrados en el Consejo Regional de Farmacia de Piauí (CRF-PI). La información fue recolectada y analizada mediante el programa Microsoft Office Excel® 2013. Los resultados revelaron que el $82 \%$ afirmó conocer la resolución mencionada, solo el $10 \%$ mencionó actividades que no encajaban en el concepto de servicio farmacéutico. En cuanto al seguimiento farmacoterapéutico, se observó que la gran mayoría conocía la acción (79\%) y realizaba algún tipo de servicio clínico farmacéutico $(82 \%)$. Solo el $6 \%$ dijo no tener espacio adecuado dentro de la farmacia / droguería para realizar estos servicios, el $88 \%$ informó que ya hizo / está haciendo especialización y solo el 3\% declaró que estaba tomando la prescripción farmacéutica. Si bien la gran mayoría de los profesionales farmacéuticos afirman conocer la resolución No. 585/2013, no saben cómo informar realmente sobre esta resolución. Además, destaca el bajo porcentaje de profesionales que realizan prescripciones farmacéuticas. Este contexto demuestra que se necesitan acciones educativas para preparar adecuadamente a estos profesionales para el trabajo clínico.

Palabras clave: Servicios farmacéuticos; Resolución; Farmacia de la comunidad.

\section{Introdução}

Atenção Farmacêutica segundo a Organização Mundial de Saúde (OMS) conceitua como uma prática profissional que visa proporcionar aos usuários uma atenção integra em que o paciente é o principal beneficiário das ações do farmacêutico, a assistência é transversal às políticas públicas de saúde compreendendo um conjunto de atitudes que tem como objetivo obter resultados terapêuticos sustentados na qualidade de vida dos pacientes (OMS, 1986). Há, no Brasil, também uma distinção legal entre farmácia e drogaria, sendo estas últimas proibidas de atuar manipulação ou fornecer medicamentos fracionados (BRASIL, 
1973). Assim, o termo "farmácia comunitária" será utilizado, ao longo deste trabalho, em seu sentido mais amplo, incluindo drogarias, farmácia de dispensação e manipulação, públicas e privadas.

A expansão das atividades clínicas do farmacêutico ocorreu, em parte, como resposta ao fenômeno da transição demográfica e epidemiológica observado na sociedade. A crescente morbimortalidade relativa às doenças e agravos não transmissíveis e à farmacoterapia repercutiu nos sistemas de saúde e exigiu um novo perfil do farmacêutico. Nesse contexto, o farmacêutico contemporâneo atua no cuidado direto ao paciente, promove o uso racional de medicamentos e de outras tecnologias em saúde, redefinindo sua prática a partir das necessidades dos pacientes, família, cuidadores e sociedade (BRASIL, 2013).

Como uma maneira de ratificar o farmacêutico diante da sociedade e aos outros profissionais, o Brasil vem apresentando modificações positivas no cenário desta profissão. Pode ser destacada a Resolução do CFF No 585, de 29 de agosto de 2013, que regulamenta os diferentes serviços clínicos farmacêuticos, e dentre as atribuições clínicas encontram-se, o acompanhamento farmacoterapêutico, a conciliação terapêutica ou a revisão da farmacoterapia, que estabelecem e conduzem uma relação de cuidado centrada no paciente.

Nessa perspectiva, o presente trabalho teve como objetivo principal avaliar o conhecimento e condutas dos farmacêuticos atuantes sobre as atribuições clínicas instituídas pela RDC N 585/13, na cidade de Teresina, Piaú, como também averiguar o conhecimento do profissional sobre o acompanhamento farmacoterapêutico, esclarecer quais tipos de serviço farmacêutico são realizados nas farmácias em que trabalham, evidenciar se as atribuições clínicas realizadas são realizadas em local apropriado, revelar a participação dos entrevistados em algum programa de Pós-Graduação como forma de aprimoramento de seus conhecimentos.

\section{Metodologia}

Trata-se de um estudo de campo descritivo, transversal, do tipo levantamento de dados no município de Teresina, localizado no Centro-Norte piauiense, tendo uma população atual de 861.442 pessoas habitantes (IBGE, 2018). O estudo compreendeu 200 farmacêuticos do município de Teresina, devidamente inscritos no Conselho Regional de Farmácia do Estado do Piauí (CRF-PI), o que corresponde a $28 \%$ dos farmacêuticos ativos perante o CRF-PI. Considerando que havia aproximadamente 714 profissionais farmacêuticos ativos no Estado do Piauí, dado este disponibilizado pelo CRF-PI em 2014.

O estudo foi dividido em duas etapas. Na primeira etapa, entrevistaram-se 100 farmacêuticos que atuam em drogarias, laboratórios de análises clínicas, farmácias magistrais, farmácias hospitalares e docentes do estado do Piauí, por meio de entrevistas por telefone, no período de 06 de outubro a 28 de novembro do ano de 2014.

Na segunda etapa, efetivou-se através de entrevistas de mais 100 farmacêuticos nos estabelecimentos das farmácias comunitárias no período de 30 de julho a 15 de agosto do ano de 2015.

No primeiro grupo, a seleção dos entrevistados foi realizada aleatoriamente em uma lista disponibilizada pelo CRF-PI, sob termo de responsabilidade de sigilo dos dados e uso exclusivo do estudo proposto, no qual, as entrevistas foram feitas através de um questionário semiestruturado contendo cinco perguntas de caráter objetivo, tendo como fonte a Resolução nº 585 de 29 de agosto de 2013 que dispõe sobre as atribuições clínicas do farmacêutico. As perguntas contemplavam a área de atuação dos profissionais, o conhecimento da RDC 585/13, serviços farmacêuticos, e o entendimento do acompanhamento farmacoterapêutico, além de avaliar o posicionamento a favor ou contra acerca da prescrição farmacêutica (Quadro 1).

O Quadro 1 mostra o questionário semiestruturado utilizado na entrevista dos profissionais farmacêuticos de diferentes áreas de atuação. 
Quadro 1 - Questionário semiestruturado para entrevista dos profissionais farmacêuticos das áreas atuantes no Estado do Piauí. Qual sua área de atuação?

Você tem conhecimento sobre a RDC n585/2013, que trata das atribuições clínicas do farmacêutico?

Cite alguns serviços farmacêuticos do seu conhecimento.

Defina acompanhamento farmacoterapêutico.

Você é contra ou a favor da prescrição farmacêutica?

Fonte: Dados da pesquisa (2021).

O segundo grupo, foi avaliado através de um questionário contendo nove perguntas sobre a mesma Resolução vigente das condutas clínicas, a respeito da realização de algum tipo de serviço clínico, bem como os serviços de apoio, ainda sobre a documentação desses serviços, o local, o conhecimento e capacitação desses profissionais (Quadro 2).

O Quadro 2 expõe as perguntas as quais foram submetidos apenas o grupo de farmacêuticos comunitários entrevistados.

Quadro 2 - Questionário semiestruturado para entrevista dos profissionais farmacêuticos comunitários da cidade de Teresina-PI.

Você realiza algum tipo de serviço clínico farmacêutico? Quais?

Você realiza algum tipo de serviço de apoio ao atendimento farmacêutico em seu ambiente de trabalho?

Caso a resposta 2 for afirmativa. Quais os serviços de apoio que você realiza em seu local de trabalho?

Caso a resposta 2 for negativa. Por que esse serviço de apoio não é realizado?

Os serviços de apoio e os serviços clínicos realizados são documentados

Você tem conhecimento sobre a resolução 585/2013?

Seu local de trabalho oferece espaço adequado para realização de serviços clínicos e serviços de apoio farmacêuticos?

Você já fez ou faz, algum tipo de atualizaçãolespecialização sobre essa área?

Realiza prescrição farmacêutica?

Fonte: Dados da pesquisa (2021).

A seleção dos sujeitos da pesquisa foi aleatória, foram abordados farmacêuticos de zonas diferentes da cidade no qual, o critério de inclusão para participação foi: ser farmacêutico auxiliar ou responsável técnico pela farmácia no Município de Teresina - PI. Como critérios de exclusão, foi estabelecida a recusa em participar.

As informações foram coletadas e analisadas por meio do programa Microsoft Office Excel® 2013 e apresentados em gráficos e tabelas digitados para um melhor entendimento em uma planilha eletrônica.

\section{Resultados e Discussão}

O conhecimento dos farmacêuticos sobre a Resolução n $n^{\circ} 585 / 2013$, foi correlacionado com suas respectivas áreas de atuação, onde $82 \%$ afirmaram conhecer a legislação referida, e desses 87,50\% atuam na área hospitalar, 75\% são docentes, 60\% atuam em drogarias e $40 \%$ e $20 \%$ em farmácias magistrais e análises clínicas, respectivamente (Gráfico 1). 
Gráfico 1 - Classificação quanto à área de atuação do farmacêutico em diferentes especialidades.

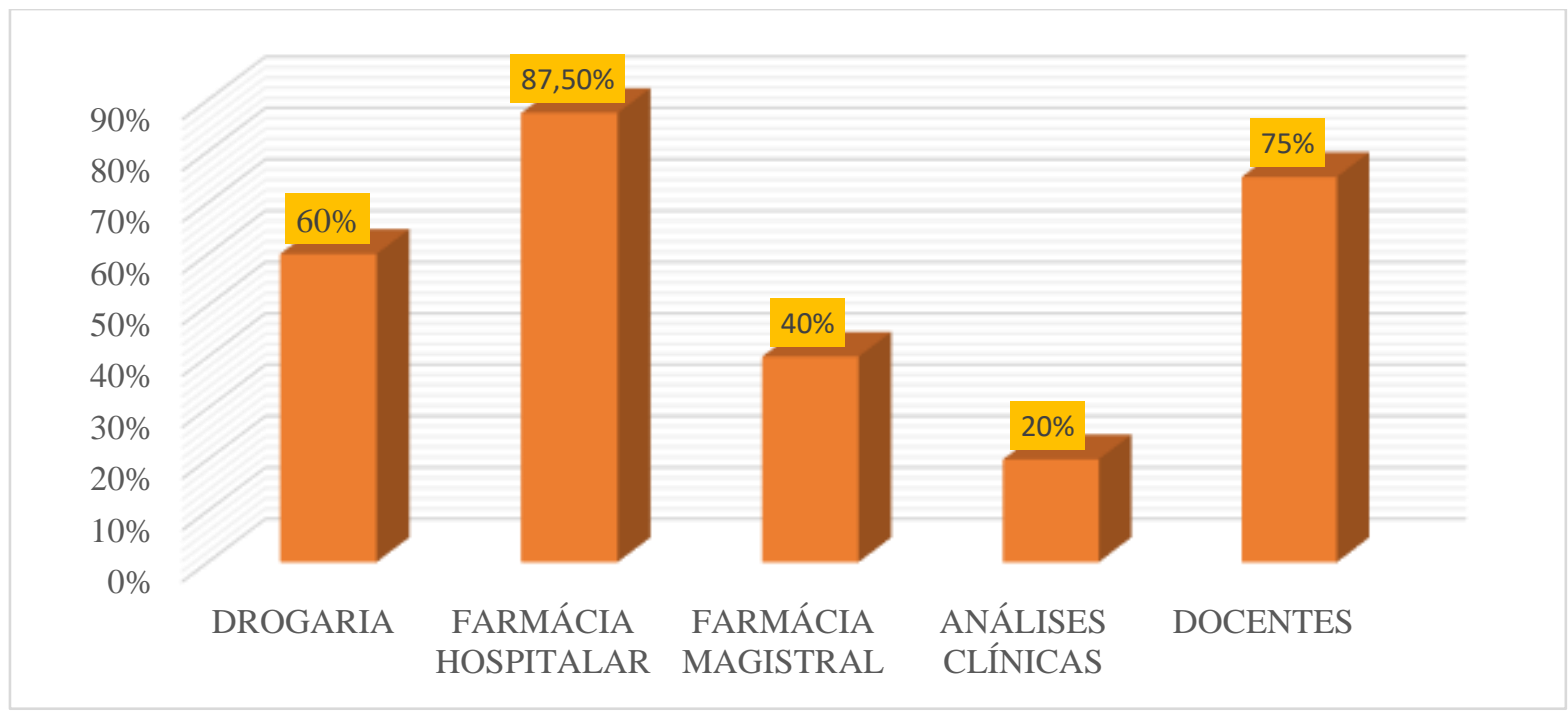

Fonte: Dados da pesquisa (2021).

Na primeira etapa, os farmacêuticos entrevistados foram distribuídos conforme sua área de atuação, drogarias (60\%), análises clínicas (20\%), farmácia hospitalar (87,50\%), farmácia magistral (40\%) e docentes (75\%).

$\mathrm{O}$ fato de o maior percentual dos profissionais do estudo terem conhecimento da resolução abordada pertencerem aos serviços hospitalares (87,50\%), pode ser explicado pelo surgimento da "Farmácia Clínica", em um primeiro momento, em âmbito hospitalar e mais tarde no ambulatório e comunidade. Menciona-se, também, que o desenvolvimento dessa área foi impetuoso nos Estados Unidos e teve um enorme impacto sobre a educação, garantindo a imagem do farmacêutico voltada ao cuidado ao paciente no ambiente hospitalar. Esse fato influenciou a introdução dessa abordagem no Brasil. Assim, foi priorizado atividades que garantissem o uso seguro e adequado dos medicamentos (COSTA, 2014).

Entre os serviços farmacêuticos mais citados pelos profissionais entrevistados está à aferição de pressão, glicemia capilar, perfuração do lóbulo auricular e outros, outro ponto relevante é que estes dados mostram que apenas $10 \%$ dos entrevistados citaram atividades que não se enquadravam no conceito de serviço farmacêutico contido na RDC 585/2013 (Gráfico 2).

Gráfico 2 - Frequência, expressa em percentual, dos serviços farmacêuticos citados pelos entrevistados.

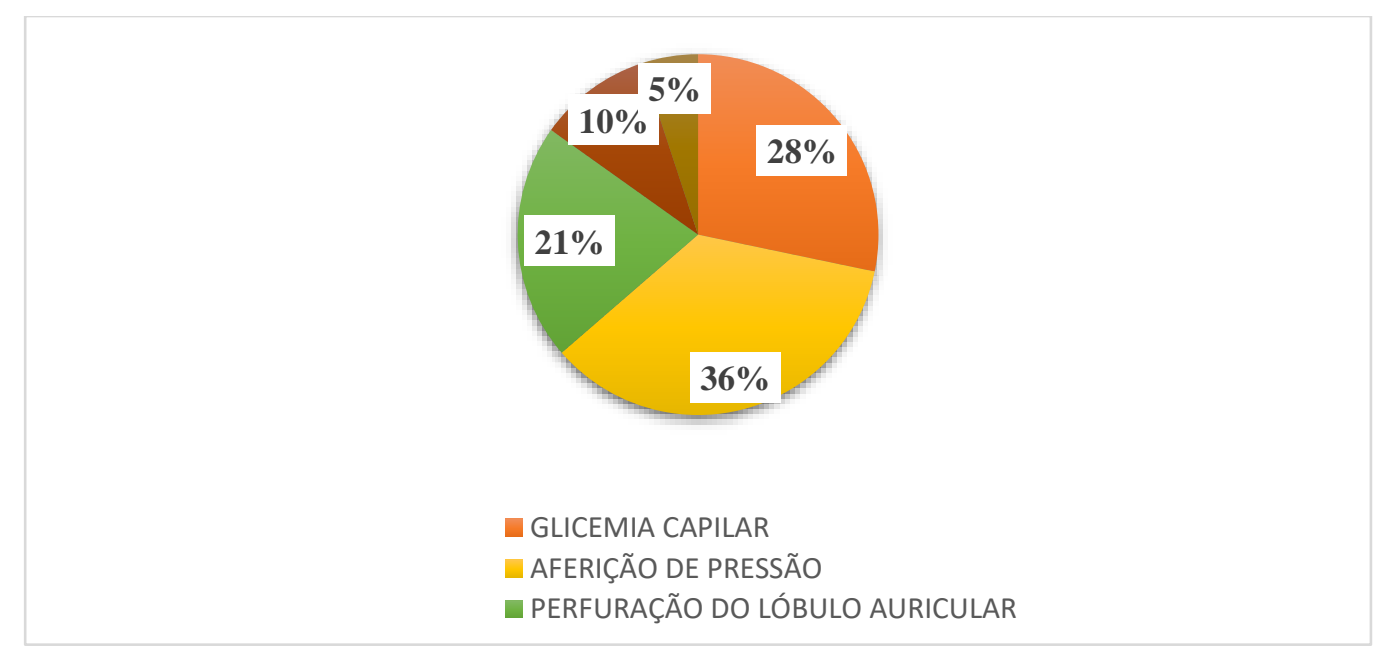

Fonte: Dados da pesquisa (2021). 
Diante disso, os serviços farmacêuticos que foram realizados de acordo com a RDC 585/2013, foram possíveis analisar que uma grande quantidade de profissionais atua realizando (35\%) aferição de pressão, (28\%) glicemia capilar, (21\%) perfuração do lóbulo auricular, (10\%) não souberem nenhum dos procedimentos realizados e (5\%) sabem aplicar injetáveis.

Fica claro, assim, que as respostas fornecidas pelos profissionais farmacêuticos estão interligadas ao real entendimento do conceito de atenção e de serviços farmacêuticos, que eles possuem, o que na verdade denota a conceituação equivocada desta prática verificando essa prática no acompanhamento ao paciente (Gráfico 3).

Gráfico 3 - Frequência relativa expressa em percentual dos principais serviços de apoio realizados por farmacêuticos na cidade de Teresina-PI.

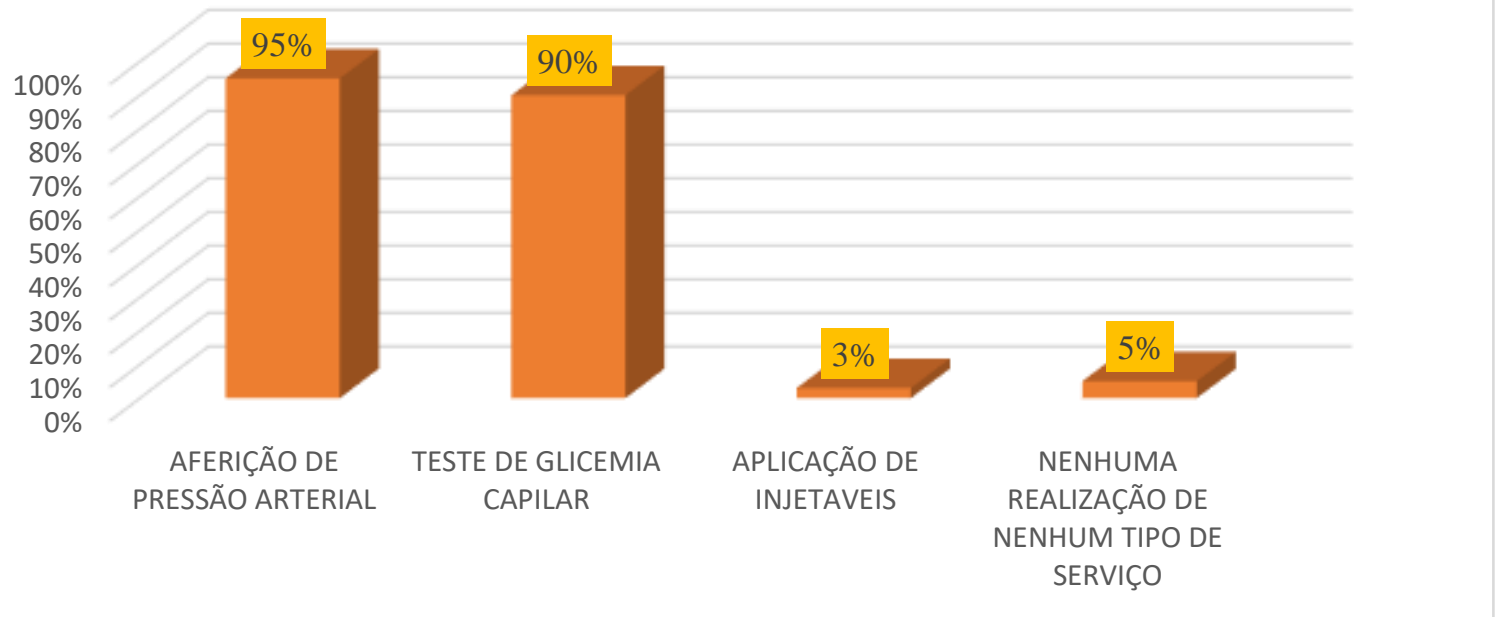

Fonte: Dados da pesquisa (2021).

Entres os serviços de apoio ao atendimento farmacêutico, $95 \%$ afirmaram realizar aferição de pressão, $90 \%$ realizam o teste de glicemia capilar, apenas 3\% registram fazer aplicação de injetáveis, $5 \%$ não realizam nenhum tipo de serviço de apoio ao atendimento farmacêutico. Sobre o método de documentar esta prática sobre a Resolução $\mathrm{N}^{\circ} 585 / 2013$ os conhecimentos dos profissionais farmacêuticos. Na Tabela 1, é possível analisar as condutas dos farmacêuticos em relação ao acompanhamento ao paciente no município de Teresina-Pi. 
Quadro 3 - Avaliação de condutas sobre o conhecimento e o acompanhamento do farmacêutico ao paciente.

\begin{tabular}{|c|c|c|}
\hline $\begin{array}{l}\text { Avaliação do conhecimento } \\
\text { profissional sobre } \\
\text { acompanhamento } \\
\text { farmacoterapêutico, expresso } \\
\text { em percentual. }\end{array}$ & $\begin{array}{c}60 \% \\
\text { Conhecem }\end{array}$ & $\begin{array}{c}40 \% \\
\text { Não conhecem }\end{array}$ \\
\hline $\begin{array}{l}\text { Avaliação do conhecimento } \\
\text { profissional sobre } \\
\text { acompanhamento } \\
\text { farmacoterapêutico, expresso } \\
\text { em percentual. }\end{array}$ & $\begin{array}{c}60 \% \\
\text { Conhecem }\end{array}$ & $\begin{array}{c}40 \% \\
\text { Não conhecem }\end{array}$ \\
\hline $\begin{array}{l}\text { Ocorrência da realização de } \\
\text { serviço farmacêutico em seu } \\
\text { ambiente de trabalho, } \\
\text { expresso em percentual }\end{array}$ & $\begin{array}{l}82 \% \\
\text { Sim }\end{array}$ & $\begin{array}{l}18 \% \\
\text { Não }\end{array}$ \\
\hline $\begin{array}{c}\text { Avaliação do conhecimento } \\
\text { dos profissionais } \\
\text { farmacêuticos sobre a } \\
\text { Resolução } \mathrm{N}^{\circ} 585 / 2013 \mathrm{em} \\
\text { Teresina-PI }\end{array}$ & $\begin{array}{c}60 \% \\
\text { Conhecem }\end{array}$ & $\begin{array}{c}\mathbf{4 0 \%} \\
\text { Não conhecem }\end{array}$ \\
\hline $\begin{array}{c}\text { Disposição de locais } \\
\text { adequados em drogarias } \\
\text { para realização de serviços } \\
\text { de apoio ao atendimento } \\
\text { farmacêutico em Teresina- } \\
\text { PI. }\end{array}$ & $\begin{array}{c}65 \% \\
\text { Apresentação local apropriado }\end{array}$ & $\begin{array}{c}35 \% \\
\text { Apresentam local não apropriado }\end{array}$ \\
\hline $\begin{array}{l}\text { Profissionais farmacêuticos } \\
\text { que fizeram/fazem } \\
\text { especialização na área clínica } \\
\text { em Teresina-PI. }\end{array}$ & $\begin{array}{c}\mathbf{8 8 \%} \\
\text { Fizeram/fazem especialização }\end{array}$ & $\begin{array}{c}12 \% \\
\text { Não fizeram/fazem especialização }\end{array}$ \\
\hline $\begin{array}{l}\text { Profissionais farmacêuticos } \\
\text { que realizam a prescrição } \\
\text { farmacêutica em Teresina-PI }\end{array}$ & $\begin{array}{c}\mathbf{3 \%} \\
\text { Realizam a prescrição farmacêutica }\end{array}$ & $\begin{array}{c}97 \% \\
\text { Não realizam a prescrição farmacêutica }\end{array}$ \\
\hline
\end{tabular}

Fonte: Dados da pesquisa (2021).

Sobre o acompanhamento farmacoterapêutico, percebeu-se que a grande maioria tinha conhecimento da atuação (60\%). Constatou-se que a maioria dos farmacêuticos se posicionam a favor da prescrição farmacêutica, porém (40\%) ressaltaram que não conhecem e não possuem especialização na área. No contexto da prescrição farmacêutica, de acordo com a Resolução $n^{\circ}$ 585/2013, trata-se do ato pelo qual o farmacêutico seleciona e documenta terapias farmacológicas e não farmacológicas, e outras intervenções relativas ao cuidado à saúde do paciente, visando à promoção, proteção e recuperação da saúde, e à prevenção de doenças e de outros problemas de saúde.

A ocorrência da realização de serviço farmacêutico em seu ambiente de trabalho, expresso em percentual de $82 \%$ dos profissionais entrevistados responderam que realizam algum tipo de serviço clínico farmacêutico em seu ambiente de trabalho. Em relação a realizar algum serviço clínico farmacêutico, $18 \%$ dos entrevistados afirmaram categoricamente não realizar nenhum. Contudo, apenas 54\% dos profissionais responderam corretamente sobre os serviços clínicos realizados, entre eles citaram-se: o acompanhamento farmacoterapêutico, a conciliação medicamentosa e a revisão da farmacoterapia do paciente e $46 \%$ afirmaram realizar serviço clínico farmacêutico. 
Dados do estudo realizado por Pontes, Nascimento e Grassi (2016), corroboraram com os desta pesquisa. Nele fica evidente que a maioria dos profissionais entrevistados acredita realizar serviços farmacêuticos, porém apenas realizam a dispensação de medicamentos, prestando instruções e orientações de modo geral e pouco específico e até mesmo automaticamente com pouca ou nenhuma interação com o paciente.

Avaliação do conhecimento dos profissionais farmacêuticos sobre a Resolução $N^{\circ}$ 585/2013 em Teresina-PI foi possível documentar esta prática, 100\% dos farmacêuticos responderam que sim e $60 \%$ dos profissionais foram contundentes ao dizer quem tem conhecimentos sobre a resolução referida. A proporção semelhante foi encontrada no trabalho realizado por Soares e Teixeira (2016), em que dos 158 farmacêuticos que disseram "conhecer completamente ou parcialmente", sobre as serem questionados sobre o conhecimento da prescrição farmacêutica, 47 (29,6\%) afirmaram não terem lido as Resoluções $\mathrm{N}^{\circ}$ 585 e 586 de 2013 do CFF. Corroborando, assim, com os dados encontrados no presente estudo que foi realizado um aspecto sobre o profissional farmacêutico em relação ao seu ambiente de trabalho em drogarias na realização de serviços de apoio ao atendimento farmacêutico em Teresina-PI.

Nesse sentido, os profissionais farmacêuticos tinham um local de trabalho com espaço adequado para a realização dos serviços citados 65\% afirmaram que tinham um local apropriado de trabalho e apenas $6 \%$ afirmaram não ter espaço adequado dentro da farmácia/drogaria que trabalham. No entanto, através da análise do estudo de Pontes, Nascimento e Grassi (2016), observou-se a discordância nesses dados em que a maioria dos estabelecimentos estudados não contavam com um espaço próprio/adequado para a realização de serviços farmacêuticos, tendo em vista que o ambiente deve ser exclusivo para o desenvolvimento da atenção farmacêutica e essencial para um trabalho de qualidade.

Nessa perspectiva, observa-se em relação aos profissionais farmacêuticos que fizeram/fazem especialização na área clínica em Teresina- Pi à realização de especializações ou atualizações na área clínica, 88\% dos entrevistados informaram que já fizeram ou fazem e $12 \%$ não fizeram ou fazem especialização. No entanto, esse perfil de profissionais não se repete em outros estados do país. Em um estudo feito por Farina e Romano-Libier (2008) realizado com farmacêuticos responsáveis técnicos por farmácias e drogarias do município de Jundiaí-SP, mostrou que 25\% dos 91 profissionais entrevistados relataram ter cursado ou estar cursando pós-graduação do tipo lato sensu.

Outro estudo realizado por Pontes, Nascimento e Grassi (2016), em que foi feito com farmacêuticos responsáveis técnicos de 15 drogarias do município de Mirassol d Oeste. Nele apresentou uma porcentagem de apenas 12\% desses profissionais buscando especializações. Contrapondo, assim, com os dados encontrados nesse estudo.

Todavia, é importante lembrar que a atualização constante de conhecimentos na área farmacêutica pode contribuir significativamente em melhores caminhos para seguimentos farmacoterapêuticos eficazes, prevenção de danos e reações, atendendo da melhor maneira as necessidades do usuário de medicamentos o que resulta em um profissional melhor capacitado a promover a atenção farmacêutica de qualidade, principalmente em Drogarias onde a automedicação é constante fazendo-se necessário maior cautela e acompanhamento por parte do farmacêutico (PONTES, NASCIMENTO \& GRASSI, 2016).

Nesse sentido, observou-se o profissional farmacêutico em relação a realização e não realização da prescrição farmacêutica com relação ao questionamento a realização da prescrição farmacêutica, somente $3 \%$ do total de entrevistados disseram realizar essa atividade e $97 \%$ não realizam essa prática. A baixa adesão à prescrição farmacêutica por esses profissionais foi percebida, também, em um estudo realizado por Soares e Teixeira (2016) feito com 159 farmacêuticos responsáveis por farmácias e drogarias de dispensação, localizadas no Município de Maringá, Paraná, em que somente 34,0\% realizavam a prescrição farmacêutica; no entanto, a maioria $(73,7 \%)$ fazia acompanhamento dos pacientes.

O elevado número de farmacêuticos que ainda realizam a indicação farmacêutica e a baixa adesão à prática da prescrição farmacêutica parece mostrar que o ato de documentar a intervenção feita na terapia farmacológica e não farmacológica intimida o profissional, mostrando que este não tem segurança para prescrever (SOARES \& TEIXEIRA, 2016). 
O estudo mostrou um cenário bastante interessante frente aos cuidados farmacêuticos, tema que existe já a um bom tempo, mas que somente nos últimos anos que está sendo mais valorizado e realizado no Brasil.

Com a necessidade de cuidados de saúde mais centrados no paciente e na melhoria da sua qualidade de vida, surgiuse os cuidados farmacêuticos. Na primeira definição sobre os cuidados farmacêuticos, referia-se que era um cuidado como um todo em que um doente requer e recebe, assegurando o uso seguro e racional do medicamento. Os pais dos cuidados farmacêuticos, Hepler e Strand, definem cuidados farmacêuticos como sendo toda a prestação de cuidados que envolve a dispensa dos medicamentos ao doente, com o principal objetivo de obter resultados no tratamento que melhorem a sua qualidade de vida. O farmacêutico tem assim responsabilidade na diminuição e prevenção da morbimortalidade associada ao uso do medicamento, assumindo um papel incontestável na saúde pública. O conhecimento regular sobre a legislação profissional e sanitária pode sinalizar um problema de atualização por meio de educação permanente referente à assistência farmacêutica em drogarias, podendo comprometer a qualidade do serviço prestado ao consumidor no que tange a promoção do uso racional de medicamentos.

\section{Conclusão}

O estudo revela que a grande maioria dos profissionais farmacêuticos entrevistados afirmam ter conhecimento da resolução $n^{\circ} 585 / 2013$, contudo ao serem confrontados com perguntas sobre a temática demonstram ausência no entendimento sobre esta resolução. Esse contexto denota a conceituação equivocada desta prática. Assim, são necessárias ações educativas e intervenções que fortaleçam essa prática.

A maioria dos profissionais declara realização de serviços e demais condutas clínicas, bem como o conhecimento sobre acompanhamento farmacoterapêutico. No entanto, ficou evidente o baixo percentual de profissionais que realizam prescrições farmacêuticas.

O estudo demonstrou ampla adesão profissional em qualificação profissional, principalmente no que diz respeito à pósgraduação do tipo lato sensu. Em contrapartida, revelou baixa adesão na realização da prescrição farmacêutica. Esse dado sugere insegurança nos profissionais farmacêuticos ao prescrever, registrar por escrito uma indicação terapêutica. Isso é fruto da desvalorização histórica da profissão.

Assim, somente com aprendizado permanente e contínuo sobre a legislação profissional, que o farmacêutico realizará com eficiência as atribuições clínicas que lhe foram concedidas.

\section{Referências}

Costa, L. S. (2014). Atuação do farmacêutico em unidade de terapia intensiva: impacto da Farmácia Clínica no acompanhamento da terapia medicamentosa. São Paulo. Dissertação de Mestrado. Universidade Estadual de Campinas.

Lucchetta, R. C., \& Mastroianni, P. C. (2010). Avaliação do conhecimento e das condutas dos farmacêuticos, responsáveis técnicos por drogarias. Rev Ciênc Farm Básica Apl.

Baldon, J. P., Correr, C. J., Melchiors, A. C., Rossignoli, P., Fernandez-Llimos, F., \& Pontarolo R. (2006). Conhecimento e atitudes de farmacêuticos comunitários na dispensação de medicamentos para gestantes. Pharmacy Practice, 4(1): 37-42.

Reis, T. M, Campos, (2013). Conhecimento e condutas dos farmacêuticos para a dispensação de medicamentos e realização da Atenção Farmacêutica em drogarias. $89 \mathrm{f}$, Dissertação de Mestrado apresentada ao Programa de Pós-graduação em Assistência Farmacêutica da Universidade Federal do Rio Grande do Sul.

Silva, D. Á. M., Mendonça, S. de A. M., Oliveira, D. R. de, \& Chemello, C. (2018). A prática clínica do farmacêutico no núcleo de apoio à saúde a família. Trabalho, Educação e Saúde, 16(2), 659-682. doi:10.1590/1981-7746-sol00108

Nascimento Júnior, B. J., Tínel, L. O., Silva, E. S., Rodrigues, L. A, Freitas, T. O. N., Nunes, X. P., Amorim, E. L. C (2016). Rev. Bras. Pl. Med., 18(1), 57-66.

Alencar, T. de O. S., Machado, C. S. R., Costa, S. C. C., \& Alencar, B. R. (2014). Descarte de medicamentos: uma análise da prática no Programa Saúde da Família. Ciência \& Saúde Coletiva, 19(7), 2157-2166. 10.1590/1413-81232014197.09142013 
Research, Society and Development, v. 10, n. 13, e102101321051, 2021

(CC BY 4.0) | ISSN 2525-3409 | DOI: http://dx.doi.org/10.33448/rsd-v10i13.21051

Hernandez-Santiago, V., et al. Alterações na resistência entre a bacteremia coliforma associada a uma intervenção antimicrobiana da atenção primária: Um estudo de série temporal interrompida por população. Remédio PLoS. 2019,16(6):1-10.

Bathoorn, E., et al. (2017). Dados da vida real sobre prescrição de antibióticos e diagnósticos de cultura de escarro em exacerbações agudas de DPOC na atenção primária. Revista internacional de doença pulmonar obstrutiva crônica. 2017,12(1):1-10.

IBGE. Instituto Brasileiro de Geografia e Estatística. Censo demográfico: Brasil, 2018. Rio de Janeiro: IBGE. < https://www.ibge.gov.br/cidades-eestados/pi/teresina.html?>.

Pontes, G. O. G., Nascimento, R., \& Gassi, L. T. (2016). Perfil e Avaliação da atenção farmacêutica em drogarias do município de Mirassol Doeste - MT. Mirassol Doeste: Revista Saberes da FAPAN. 3(1), 13-25.

Organização Mundial da Saúde. (1986). Carta de Ottawa. Em: Promoção da saúde e Saúde Pública (P.M. Buss, org.), 158-162, ENSP.

Conselho Federal de Farmácia. (1973). Lei no 5.991, de 17 de dezembro de 1973. Dispõe sobre o controle sanitário do comércio de drogas, medicamentos, insumos farmacêuticos e correlatos, e dá outras providências. MS.

Conselho Federal de Farmácia. (2013). Resolução CFF n585, de 29 de agosto de 2013. Regulamenta as atribuições clínicas do farmacêutica e dá outras providências.

Soares, A. L. P. P. P., Costa, M. A., \& Teixeira, J. J. V. (2016). Nível de entendimento sobre prescrição farmacêutica no Brasil. Estamos preparados para essa nova realidade? Rev. Informa. 149-156.

Vosgerau M. Z. S., Souza, R. K. T., \& Soares, D. A. Utilização de genéricos em área de atuação da equipe de saúde da família em município do sul do Brasil. Rev Bras Epidemiol 2011, 14:253-63. 